THE INFLUENCE OF PROLAPSE OF THE KIDNEY ON 'I'HE PRODUCTION OF DISEASE OF THE FEMALE PELVIC ORGANS.*

AUGUSTIN H. GOELET, M.D.

Professor of Gynecology in the New York School of Clinical Medi. clne ; Gyneculogical Surgeon to the Metropolitan Hospital for Women and Childrev, Etc. NEW YOJKK CITY.

Prolapse of the kidney may cause or maintain discase of the female pelvic organs by interfering with the return circulation from the pelvis and pioducing pelvic congestion. This may be brought about chiefly in two ways:

1. The renal artery being less yielding than the other structures constituting the renal pedicle, dragging upon it by the kidney crowds the aorta over against the vena cava and distorts that great channel of return circulation when it is the right kidney that is prolapsed.

2. When the displaced kidney reaches a certain point in its descent it is situated immediately in front of the ovarian vein as it ascends to empty into the vena cava just below the renal vein on the right or into the left renal vein on the left side. Hence, pressure backward upon the kidney, as from corsets, or clothing at the waist, or from accumulation of gas or feces in the colon, would cause it to compress the ovarian vein or veins against the solid structures of the back and obstruct the circulation. It must be remembered that valves are rarely found in these veins, therefore the circulation is more readily interfered with.

Even in the early stages of prolapse, the kidney may compress the ovarian vein, because it does not, as a rule, descend directly downward, but more often the lower pole is directed inward toward the spine and overlaps the vein and ureter. (See figure.)

There is no doubt that the circulation of the ovarian vein may also be arrested by sharp flexure of the ureter over it when the kidney is in an advanced degree of prolapse, since the ureter is behind the vein where they cross and is fixed more or less by the peritoneum covering it.

Another cause of compression of this vein, at times. may be distention of the ureter when for any reason there is obstruction below the point where it is crossed by the vein.

If this obstruction to the circulation through the ovarian vein was constant, there would undoubtedly be established an accommodation for the return circulation from the pelvis through other channels, but since it is not constant, the effect is more pronounced and the result is a profound congestion of the pelvic organs, under favorable conditions, when the kidney is prolapsed; that is, during the greater part of the twentyfour hours when the patient is about on her feet.

This cause of compression of the ovarian vein by the kidney is not observed in autopsies because the kidney is found replaced usually; unless it has become fixed in its abnormal position by inflammatory action involving the structures immediately surrounding it. At any rate, the exaggerated degrees of prolapse possible during life do not, as a rule, prevail after death.

The hepatic and intestinal disturbances associated with prolapse of the kidney also have a marked influence upon the production of pelvic congestion.

Prolapse of the kidney may not, in every instance,

* Read at the Fifty-third Annual Meeting of the American Medical Association, in the Section on Obstetrics and Diseases of
Women, and approved for publication by the Executive Committee : Drs. A. H. Cordier. W. E. B. Davis and Fenry P. Newman. produce pelvic congestion because the position may be such that it does not compress the ovarian vein, or the kidney may be unusually small-atrophied-and may not occupy sufficient space to make this result possible, or the other conditions enumerated may not prevail. Then, too, all women do not wear corsets or tight clothing. It must be remembered, however, that the prolapsed kidney is frequently larger than normal from vascular engorgement, because traction upon the renal pedicle incident to prolapse narrows the caliber of the renal vein by stretching it out, thus retarding the return circulation from the kidney, or it may result from twisting of the renal pedicle.

Atrophy of the prolapsed kidney may be produced by prolonged pressure from accumulated urine in the pelvis and calices from obstruction of the ureter due to flexion or torsion, or calculus. Yet these atrophied kid-

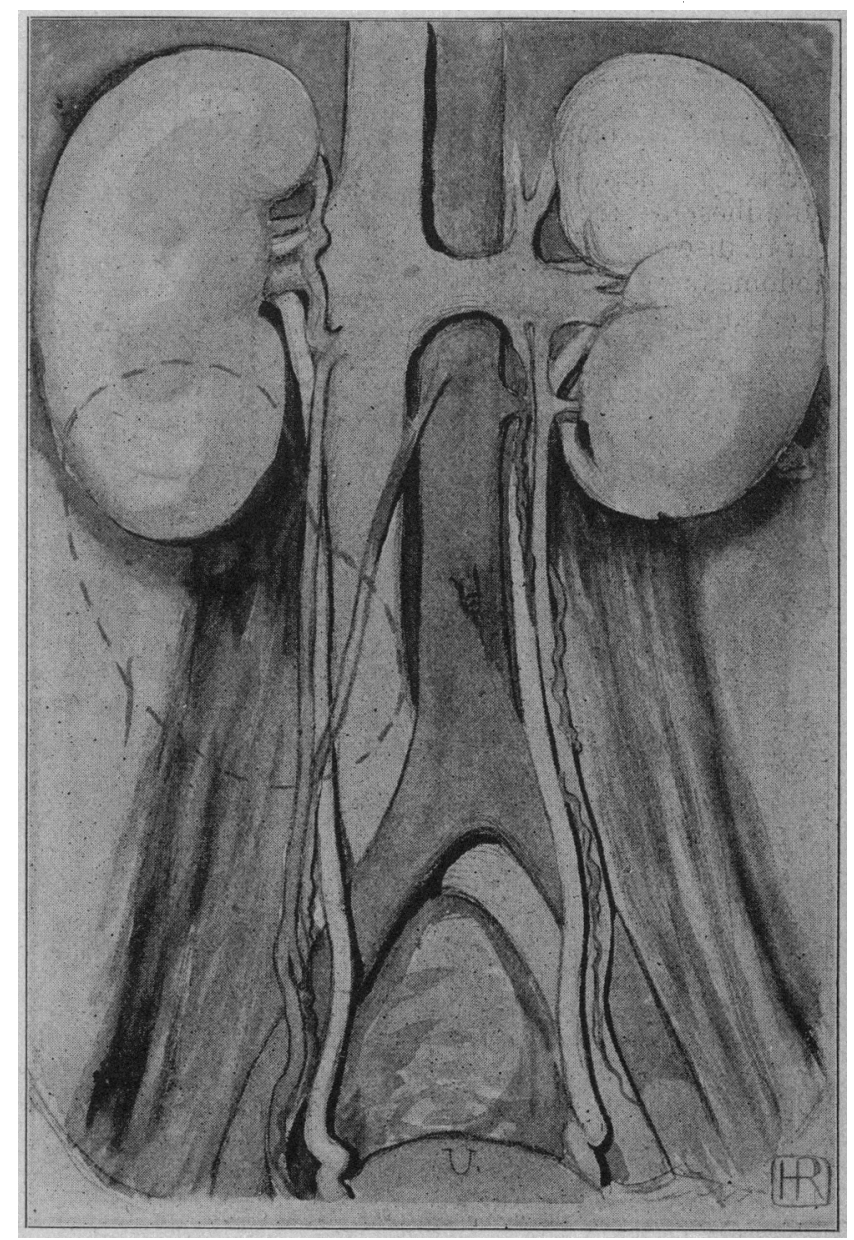

The dotted outline shows the position of the kidney when prolapsed and illustrates how it may compress the ovarian vein and ureter when the abdomen is compressed by corsets or clothing.

neys may at times become greatly enlarged from vascular turgescence or hydronephrosis (H. Morris). Hence, the same kidney may be unusually small at one time and greatly enlarged at another. It is well to hear this in mind in estimating the possible influence a small misplaced kidney may have upon the circulation if the pelvis.

CONDITIONS IN THE PELVIS PRODUCED BY PROLAPSE OF THE KIDNEY.

The conditions in the female pelvis that are found most frequently associated with prolapse of the kidney and apparently dependent upon it as a cause. either direct or contributing, are: 
Dysmenorrhea of the congestive type, the hyperemia involving the tubes and ovaries as well as the uterine mucosa. When brought about by this cause, the dysmenorrhea is usually acquired after puberty and increases in severity, resisting the measures that ordinarily afford relief.

Profuse, persistent leucorrhea of nonseptic character I have often observed in conjunction with prolapse of the kidney. It appears to be a hypersecretion due to simple hyperemia and is most intractable, causing great discomfort to the patient. It is very often encountered in unmarried women who have one or both kidneys prolapsed, and it disappears only when the kidney is fixed.

Endometritis of the so-called catarrhal variety, salpingitis of the same type, and ovaritis with, at times, considerable enlargement of the ovary, are often found associated with prolapse of the kidney. In this connection I recall a case sent to me for operation by the family physician with a diagnosis of pus tube and endometritis. On examination I found a large right ovary which was very sensitive, and the right kidney prolapsed to the fourth degree. There was also a laceration of the cervix and a profuse discharge. There were, however. no adhesions about the ovary and no evidence of structural disease. I decided, therefore, not to open the abdomen, but curetted, repaired the cervix and fixed the kidney. The patient made a perfect recovery. The orary gave no further trouble and soon became reduced to normal size.

Endometritis associated with prolapse of the kidney and depending upon it as a cause, are the cases so often encountered that have been submitted to repeated curettage and remain uncured. I have on my record book a number of cases of this kind where a cure has been finally effected by fixing the kidney.

Endometritis and salpingitis of a more serious type may also result from this same cause, because such a condition of the uterine mucosa as would result from persistent hyperemia due to prolapse of the kidney affords a fertile field for the development of pyogenic bacteria introduced from the ragina and cervix which would gain no foothold in its absence.

Uterine displacements often result from increased weight of the uterine body due to prolonged congestion, which also weakens the supports, and hence may be brought about by prolapse of the kidney. Displacements of the uterus in unmarried women and in young married women who have never been pregnant may be accounted for by prolapse of the kidney acting as a cause. It is certainly remarkable how many women of this class have posterior displacement of the uterus and prolapse of one or both kidneys. The following case affords a very good illustration. A young woman, eight months married, upon whom I operated for prolapse of the right kidney, would not consent to shortening of the round ligaments at the same time for a heavy retroflexed uterus, though it was urged. I, however. curetted the uterus for a catarrbal endometritis, replaced the uterus and inserted a pessary at the same time that the kidney was fixed. She had never been able to wear a pessary for any length of time before, though it had been tried at different times. The pessary inserted at the time of the operation was retained while she was in bed without her knowledge, and for two weeks after she was up-five weeks in all. When she discovered it she insisted upon having it removed, because the knowled re of its presence in the vagina annoyed her. The uterus remained in position after it was removed and did not again become displaced. I must credit the cure of the displácement in this case to fixation of the kidney, and must think the maintaining cause was displacement of the kidney, since a cure could not have been effected by curettage and a pessary alone worn for five weeks.

Hemorrhage from the uterus-menorrhagia and metrorrhagia-is another condition often found associated with prolapse of the kidney without other ascertainable cause. In two cases that came under my observation, it had resisted previous curettage, recurring after a few months, but it ceased after curettage combined with fixation of the kidneys.

Hematoma and hematocele not the result of ectopic gestation may be produced by over-distention of the ressels of the broad ligaments, having its origin in prolapse of one or both kidneys. I have the records of several such cases. In one of my cases where I opened, the abdomen at the same time after fixing both kidneys, I found a hematoma of the right ovary. This ovary and that on the other side were both buried in adhesions, though the tubes were in good condition and presented no evidence of the inflammation having extended from the uterus.

Cystitis is also observed in conjunction with prolapse of the kidney, and may be caused by infection from the kidney or may result from prolonged congestion due to the position of the kidney. Irritable bladder is certainly a frequent symptom, being present, according to my experience, in about 15 per cent. of these cases.

There may be other conditions in the pelvis that I have not observed, traceable to descent of the kidney as a cause, and it is well to bear this possible cause in mind in dealing with those conditions which may be brought about by prolonged pelvic congestion. I would urge, therefore, that as a routine measure all gynecological patients be examined for prolapse of the kidney.

\section{CLINICAL SIGNIFICANOE.}

The clinical significance of nephroptosis is well illustrated by the diversity of the symptoms it gives rise to. These may be arranged in three groups:

1. Gastro-intestinal disturbances, manifested by gastric irritability. intestinal distention, constipation, repeated bilious attacks and recurring attacks of jaundice

2. Disturbances of the circulation and of the nervons system, manifested chiefly by vertigo, syncope, palpitation of the heart, epigastric pain, neurasthenia, hysteria, nervousness, restlessness, insomnia and lassitude.

3. Disturbances of the genito-urinary organs, manifested by symptoms of disease of the female pelvic organs, irritability of the bladder and paroxyms of renal pain, which may be due to obstruction of the ureter from flexure or twisting, or to stone in the calices, pelvis or urreter.

Pain in the region of the kidney is rare. Apart from the renal crises referred to, it is usually limited to a dull aching in the loin, sometimes extending to the groin and down the thigh. Very often these patients fail to mention pain in this location when enumerating their symptoms, but will admit it when pressure upon the sensitive displaced kidney serves as a reminder.

Pain in the region of the appendix and in the ovarian region of the same side as the displaced kidney is not uncommon.

Loss of flesh sometimes amounting to emaciation is often observed, and in some of these cases there is pronounced-anemia, showing that prolapse of the kidney may produce a profound impression upon the general srstem, and unless it is correctly accounted for it would not be remedied. I have known some of these patients 
to lose as much as 50 or 60 pounds within a period of two years. As a rule, the influence of nephroptosis upon the general system is pronounced only when the degree of prolapse is exaggerated or when the function of the kidney is seriously interfered with and the organ has become diseased.

Unfortunately the symptoms are seldom of sufficient severity to be unbearable, which leads to neglect of the condition even after attention has been directed to it. Many times the symptoms appear to be absent when the prolapse is detected because no effort is made to bring them out in the history; hence it is often asserted that nephroptosis does not, in some instances, produce symp. toms, but if the examiner is familiar with the symptoms and looks for them, he will find them.

\section{INFLUENCE OF PROLAPSE ON THE KIDNEY.}

The influence of prolapse upon the kidney itself is, at times, very pronounced. It is unfortunate, however, that this is not discovered in many cases until the kidney is exposed on the operating table. But there are some grave conditions which may be detected by careful observation upon external examination, and others that are revealed by microscopic. examination of the urine if the examinations are conducted by an expert.

The possibility of prolapse of the kidney leading to structural disease of the organ is apparently ignored. Why this should be so is certainly strange. It is well understood that it interferes both with the circulation and function of the organ; hence it must lead to disease. Traction upon the renal pedicle by stretching the vein, diminishes its caliber and restricts the return circulation, producing venous engorgement or congestion. This effect is sometimes well illustrated when the kidney is drawn out upon the surface of the back through the lumbar incision. Descent of the kidney or change of its position may produce obstruction of the ureter by sharply bending it or by twisting the renal pedicle, and thus the outflow of the excreted urine is arrested. In consequence it accumulates in the pelvis, distends it and the calices, and retards or suspends the work of the kidney. When this distention is prolonged it results in atrophy.

If prolapse does not always produce obstruction in the manner just described, urine may accumulate in the pelvis because of the position of the kidney below the point of flexure of the ureter, or the ureter may become compressed by the kidney which overrides it, in the same manner as the ovarian vein is compressed.

The conditions then that may be produced by prolapse are those that would result from congestion of the organ, or obstruction of the ureter; hence, the prolapsed kidney may be affected by nephritis, perinephritis and pyelonephritis, hydronephrosis and pyonephrosis, or atrophy may result. Perinephritic blood extravasations and extravasations under the fibrous capsule are observed also on the operating table in cases of nephroptosis of long standing.

When prolapse of the kidney comes to be regarded as a serious condition which requires rectification, disease of the kidney will be a more rare affection, because these conditions will be forestalled by early operation.

THE REMEDY: ITS JUSTIFICATION AND APPLICATION.

This leads up to the question: When does prolapse of the kidney require operation for its rectification, and when may it, with impunity, be treated by palliative measures?

It is now a well-established fact that belts and supports of all kinds for the kidney are not only valueless, but positively harmful in advanced degrees of prolapse where they would be most needed. They not only fai] to afford proper support for the kidney, but provoke irritation by the pressure they exert upon it. They are very often discarded by patients because they produce discomfort and pain in the region of the kidney, though they may have afforded relief of the symptoms at first. Hence, the more efficient the support the more objectionable it is.

It has been shown that even the manipulations of the kidney incident to diagnostic palpation produce irritation, evidence of which is often to be found in the urine excreted after these manipulations. How much more objectionable, then, would be the irritation provoked by a pad pressing constantly upon the kidney. A snug-fitting belt is only a little less objectionable. This obtains, however, only in advanced degrees of prolapse when the kidney has descended so that its upper pole comes below the lower border of the last rib in front (third and fourth degrees).

When the kidney is prolapsed only to the first or second degree, belts are of use in supporting it and preventing or retarding its descent (?). But it is most unwise to disregard this condition when it is discovered, because apparently it is not producing symptoms. The tendency of prolapse of this organ, even of minor degree, is inevitably progressive, and in time it must become exaggerated if left to itself. Hence, prolapse of the first and second degree admit of external support by belts, but pads employed for producing pressure upon the abdominal wall with the intention of supporting or replacing the kidney, are objectionable and are to be discouraged.

Prolapse of the third degree or beyond requires operation, whether it is producing symptoms or not, or whether or not there is evidence of disease of the kidney, if the disease has not advanced beyond recovery, for the reasons already mentioned, which are self-evident. If the other kidney is prolapsed only to the second degree, it should be fixed at the same time so as to avoid a second operation, which would subsequently become necessary. My experience leads me to be very positive upon this point. The operation is one of so little gravity that anyone requiring it should not be deterred from taking advantage of the opportunity it affords for cure of a condition that may be both troublesome and dangerous if neglected.

The failure of early operators to attain the degree of success desirable in securing permanent fixation of the kidney deters many from advising operation, and some surgeons even discourage it. When, however, it comes to be more generally understood that the prolapsed kidney may now be permanently fixed, without injury to its structure and without risk to the patient, the operation will be more universally urged; more especially as there is no alternative, no other positive means of cure of a condition that carries with it often not only chronic invalidism, but a constant menace to health and even life, in disease of the most important excretory organ of the body.

\section{DISCUSSION.}

Dr. G. B. MASSEY, Philadelphia-With all due deference to our colleague, I say this is dreadful. An eminent lawyer has recently called the United States Supreme Court the "court of last conjecture." How much we need such a court in medicine! Look at the basis of this conjecture that prolapse of the kidney pressing on a vein causes disease of the pelvis. See how far it is away from the pelvis; how much opportunity for pressure exists in all directions. Examine these cases and find out how many of them with pelvic symptoms 
enumerated by the Doctor have this prolapsed kidney. I have had a number of eases of prolapsed kidney and can not recall one instance where conditions in the pelvis existed simultaneously. Many cases come to the specialist complaining of uterine disease when they have a floating kidney, it is true, but rou find no symptoms of pelvic disorder; the patients merely thought they had uterine disease.

What shall be done with floating kidneys? What is the matter with these patients? Everyone of them is profoundly neurasthenic. Floating kidney is due to the absorption of its fatty capsule, because of the patient's neurotic conditionnot a brain neurosis, but an abdominal neurosis. The floating kidnev is a mere effect, not a cause. What, then, is the cause of the abdominal neurosis? Speaking from a clinical experience in the treatinent of these cases, I should say that we have abdominal neuroses in consequence of auto-intoxication. As to treatment: If you give these patients powerful abdomino-dorsal galvanic currents, 50 to 75 milliampères daily, stimulating thoroughly the abdominal circulation, the condition will be remedied. You can add to this nitro-muriatic acid, giving five drops of the full strength acid after meals, and also the compound rhubarb pills as a stimulating cathartic. I am not theorizing, but speaking of facts. Many of these cases have aortie pulsation due to the neurosis and this featme has receired but little attention. Your purpose should be to reanchor the kidney with fat, and the treatment outlined will often lo it.

JR. WAiTER P. Mastox, Detroit-I have had twenty years' experience in the treatment of these cases, and I must disagree in toto with the last speaker. Dr. Massey misunderstand, the condition. He confounds floating kidney, which is a congenital condition, with loose kidney, which is an acquired

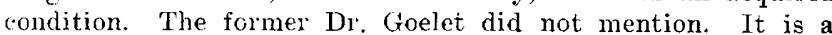
very different thing from the disorder under consideration. Roughly speaking, I presume that 40 per cent. of all the women applying for treatment of local disorders hare a loose kidney. In my own experience I have found that a large proportion of these cases with loose kidney also have pelvic troubles, frequently associated with chronic appendicitis. In a considerable number of these cases we also find gastroptosis and enteropicsis, and many symptoms point to those conditions, and not to the kidney. I believe that the only treatment of a loose kidney, when symptoms are presented, is fixation. For several years I antagonized this operation, but $I$ have since experienced a change of heart, and I now operate on every case where the kidney is prolapsed and gives rise to symptoms; especially those cases which Dr. Goelet designates as of the third and fourth degrees.

I believe that the only time when belts or pads are permissible is in the case of elderly women where operation is for some reason undesirable. A properly-adjusted belt will sometime afford the patient comfort and give relief, especially from the symptoms incident to kinking of the ureter. In younger women, however, I believe fixation is always the better procedure and most desirable.

DR. L. H. DUNNivg, Indianapolis-This is one of the most difficult questions we have to consider in relation to abdominal diseases. We have had two extreme views expressed today, but when the question is finally settled, the truth will be found to lie about midway between the two. I speak advisedly, because I was one of the pioneer workers in this line in this country. I have delved into the subject as deeply as I have been able and have had considerable experience, but I an compelled to say that my mind is still unsettled as to the influence and cause of movable kidney and the symptoms induced by it. I believe I operated on the second case of this kind in this country. At first I operated on all cases that presented themselves, doing many operations in a short space of time. I operated by the method of stitching the fatty envelope of the kidney to the muscle and fascia of the lumbar incision. About one-third of these operations were unsuccessful. The subject was new at that time, and we did not study the symptoms produced by it, but every symptom presenting itself was ascribed to the movable kidney, and in nine cases out of ten the symptoms were not relieved, even though the kidney was fixed. Then I refused to operate for a number of years, and used pads, believing that movable kidney was one of the symptoms of a general enteroptosis, and that the gastrointestinal disturbances were largely the causes of the movable kidneys, rather than the efiects, and that idea prevails in my mind at the present time.

I am disposed to agree very largely with what Dr. Míassey has said on this subject as to the influence of movable kidney on diseases of the pelvis and the abdominal organs. I do not believe the operation is dangerous. I have done anywhere from sixty to eighty of them. A man can do as many as he chooses, as such patients present themselves continually. In looking orer my notes recently, I found that about 7 per cent. of women presenting themselves to me for examination had a movable kilney. I could have operated on the majority of these had $I$ chosen to do so. I believe the danger of the operation is almost nil, providing the patient's condition is such as to permit of an operation, but the relief that comes from the operation $I$ believe to be very small in the majority of eases unless attention is also given to the relief of coincident conditions. The treatment which will relieve the coincident general enteroptosis and gastro-intestinal disease must be considered the best. The present methods of operation are very satisfactory, but in nearly erery instance of fixation we fix the kidney in the first degree of prolapsis.

Dr. Charles P. Noble, Philadelphia-I suffered the change of heart that Dr. Manton spoke of many years ago, and I have been studying loose kidneys very carefully since 1890 . I have operated on about 75 cases, but if $I$ had followed the lines laid down by Dr. Goelet, the number would have been between 1000 and 1500 . I think the Doctor takes an extreme view as to the indication for operation.

It seems to me that the cause and effect and the coincidents have been very much mixed up. It is a fact that a large number of the patients are imvalids, and that the kidner's are loose because they are thin. They also have congestive trouble involving the pelvic organs as well as the abdominal. The congestive condition is due to the general health far more than to the loose kidney. I an a very firm believer in the operation of fixation of the kidney in suitable cases, but I think it is one of the most difficult problems the surgeon has to solve to say when the kidney ought to be fixed. Kidney operations have given me as good results as any work I have ever done, but occasionally no good results are secured. It must be remembered that the symptoms may be due to hysteria or neurasthenia, and not to a movable kidney, which is a coincidence. Dr. Goelet spoke about the great danger of serious damage to the kidney. I have seen a few such cases, hydronephrosis, two cases of abscess in the kidney, which were probably predisposed to by displacement of the kidney. They were only a few out of 1500 or 2000 cases that I have seen, so that the Doctor's fear of damage to the kidney is exaggerated. Unless the patient has pain or evidences of congestion, I do not think we need fear that the kidney is going to degenerate. I believe in the operation of fixation, but I could easily do ten times as many operations as are necessary if I so desired.

Dr. Charles S. Bacon, Chicago-I am surprised at the statement that the operation for prolapse of the kidney is so far developed that it always gives good results. I think that is contrary to the experience of most operators, and that the statement can hardly be accepted at the present time. The relation between prolapse of the kidney and pelvic disturbances is not positively established, and in the majority of instances there is merely a coincidence. I believe that there are two conditions present in prolapse of the kidney; one, where it is associated with prolapse of other organs, a general splanch. noptosis; there is generally a weakness of the abdominal walls perhaps due to pregnancy, which results in a variety of symptoms that go to make up the symptom-complex of enteroptosis or splanchnoptosis. Then there is another condition in women who have not a general enteroptosis, where the abdominal walls are not particularly prolapsed, but the kidney is prolapsed, and is frequently the cause of pain, congestion and other symptoms that result from this pathologic condition. 
In the first class of cases the reasonable indication for treatment is the condition that leads to the prolapse. Strengthen the abdominal wall by systematic exercise, by movements which tend to strengthen; massage and general electricity, and in the serious cases a support or bandage, that braces up the relaxed tissues. The great trouble of the supportive treatment is that the support is often applied directly over the kidney. The abdominal contents should be supported from below by a broad bandage, or one containing pads that make pressure in an upward direction. Such treatment as that properly carried out will succeed in giving relief and curing the condition. Support of itself is harmful, unless combined with the treatment which gives an increase in the natural support, the abdominal wall.

Dr. Gustay Kolischer, Chicago-Since Dr. Edebohls said that appendicitis was caused by a floating kidney, the kidne $\vec{y}$ is held responsible for all kinds of pathologic conditions. Pathology is an exact science, bnt the explanation of cystitis occurring frem a prolapsed kidney is absolutely inexact and incorreet. If the kidneys were placed in such a position as shown by the Doctor on his diagram, we would have an acute hydronephrosis in twenty minutes. If the kidney becomes morable, does it fall back or down? I do not see how this organ can possibly compress the veins and produce pelvic disease. The essayist himself admits that in postmortems we never find the effects of engorgement from compression of the reins. That the morable kidney can not compress the ovarian rein can be found by examination of any case of floating kidney. We ean pick it up with one hand without any trouble and find it elose to the anterior abdominal wall, whereas, if it compressed the ovarian artery against the bony walls of the pelvis, we could not pick it up. The Doctor says that engorgement and impairment of circulation take place easily, because there are few ralves in the veins which have really nothing to do with engorgement, but simply hinder the reflux of the venous blood, and divide up the column of blood so as to hare it more easily propelled. He says that endometritis is produced by a floating kidney. This term is unscientific; in most cases we have to deal with a hypertrophy and not with an inflammation of the endometrium. 'Terms are used throughout the paper without any exact meaning. I should like to ask, what is a dysmenorrhea of the congestive type? I can not see what the kidney has to do with dysmenorrhea. It is a contraction by jerks, like insufficient contraction of the colon in diarrhea. If the kidney impairs the circulation of the pelvis in any way, why do not twe find edema in such cases? If the rena cava is compressed, we have edema of the limbs and abdomen.

So far as the operation is concerned, these gentlemen who adrocate it uniformly do not know the literature. Floating kidney can be cured by improving the general condition of the patient. Massage is also valuable. There are bandages on the market which will retain a floating kidney in certain cases. IIith all due respect and regard for the opinions of the gentlemen who have spoken, I must say that they run contra to all our pathologic convictions.

Dr. Rufus B. Hald, Cincinnati-The extreme views of the essayist and Dr. Massey are not without value to every thinking man. Early in my career I operated on some movable kidneys, believing that I would cure my patients, but as my patients would come back complaining of exactly the same symptoms, I commenced to question the advisability of this operation. I studied my cases more carefully, putting them to bed for three or four weeks, so as to eliminate all conditions which might be accounted for in other ways, and I was surprised how small a minority of cases was due to the falling kidney. If you operate on all cases of morable kidney of the second or third degree, many patients will have a recurrence of their simptoms and you bring surgery into disrepute.

I can not aceept the pathology given by the essayist, as I do not believe movable kidney to be the cause of all the symptoms enumerated by the essayist. It is a very valuable paper, however, and I enjoyed it very much.

Dr. EFFie Lobdell, Chicago-Corsets have been mentioned as the cause of this tronble. I think that if the gentlemen would adopt the use of the corset in the treatment of this condition it would be of considerable benefit. In a number of cases in my clinic $I$ have used a straight-front corset, having the patient put it on in the morning before rising, and it has been worn with much comfort. I have used it in serub-women, housewives and women of leisure, with benefit in each case.

Dr. J. H. Canstexs, Detroit-There are cases of loose kidney which are the result of accidents, such as a fall from a stepladder or heavy lifting and that class of patients will always get well if operated on. Those cases which are due to relaxed conditions of the abdominal wall are an entirely different class. I do not believe that the pressure produces appendicitis or ovarian trouble, but there certainly is an irritation of the sympathetic nervous system, and from this you have the real trouble of the pelvic organs.

DR. GOELET, in closing-If I have succeeded in making you think of this subject after you go away I will be satisfied that my work has not been in rain. At the next meeting you will agree with me. Take your cases and study their symptoms. I thought as many of you do when I began this work. The neurasthenic symptoms are produced by the prolapsed kidney; the loss of flesh which accompanies it is to be attributed to the same cause and is not the cause of the prolapse. I have never used electricity for this condition and do not feel inclined to do so as I fail to see any indication for it or how it could be of benefit. I did not include loose kidneys, but spoke of kidneys prolapsed with the upper border below the level of the last rib in front; prolapse of the third and fourth de grees. I only consider the lesser degrees of prolapse when operating on a prolapse of greater degree. As our Chairman has very aptly said, "if we operate on these cases early, we may cure them." Do not wait until the patient is in such a deplorable condition or the kidney is in such a bad shape that there is no chance of a cure. Operate early and you will cure a greater number. One gentleman objected to operating on patients of advanced age. I do not know what he calls advanced age. I have operated many times with satisfaction on women orer fifty. The question under discussion is not the method of fixation of the kidney; that I shall discuss tomorrow morning in a paper that 1 shall read before the American Urological Association.

There are three ehief reasons for failure of the operation to give relief: First, operating too late after the kidney has become seriously crippled; second, fixation of the kidney too low down, so that it can be pinched or compressed by the clothing or corsets at the waist, and third, failure to secure permanent and positive fixation. There are several methods of suturing the kidney and many of them result in failure. That is the reason for the dissatisfaction with the operation felt by some operators. It seems to me that the truth is to be arrived at only by. radical riews in both directions, and at the next meeting of this Association I feel confident that more of you will agree with me, provided you carefully investigate the subject in the meantime.

As to the eriticisms of my pathology, I believe the gentleman from Chicago will admit that there are many diseased conditions of the pelvis, of which pelvic congestion is the starting-point. I do mean to say most emphatically that prolapse of the kidney is one of the causes of pelvie disease and that their association is not a coincidence. The dropping down of the kidney, without any compression of the abdominal wall, I am willing to admit, would not cause compression of the ovarian vein though it might drag on the aorta and distort the caliber of the vena cava. When the kidney drops down, even in the earlier degrees of its descent, it swings around so. that the lower pole overlaps the ovarian vein and ureter. Tight clothing or the corset crowds the kidney back against the vein and compresses it and the ureter between it and the solid structures of the back. While we would not suppose that the kidney could compress the ovarian vein under normal conditions, it does do so when it is prolapsed when the patient is. on her feet and the abdomen is compressed.

As to prolapse of the kidney being a cause of cystitis, I be lieve the gentleman from Chicago will also admit that the bladder may become infected from the kidney and the kidncy 
may become infected through the circulation as the result of the prolapse. Besides, as I said in my paper, the congestion of the bladder mucous membrane maintained by prolapse of the kidney affords a favorable field for the growth of septic germs. Endometritis may develop also in the same manner because of a maintained congestion of the endometrium. The operation for fixing the kidney has no actual mortality. I have done up to this date 136 nephropexies on 109 patients, of which there were 27 double nephropexies at the same time without a death and without a single unpleasant complication following the operation.

\section{AN OPERATION FOR ESTABLISHING A CUL- DE-SAC FOR THE WEARING OF AN ARTIFICIAL EYE; WITH RE- PORT OF CASES.* JOHN E. WEEKS, M.D. TEW YOLK CITY.}

In the endeavor to establish a cul-de-sac in cases where from any cause the conjunctival sac has become reduced to such an extent that an artificial eye can not be worn, very many devices have been resorted to. Operative procedures which involve the transplanting of a flap of integument or of mucous membrane have been most numerous. Flaps of integument with a pedicle, flaps without a pedicle (Wolfe) taken from various parts of the body, Thiersch grafts, mucous membrane from the lips (Abadie), vulva ${ }^{1}$ and prepuce (Bock) using that of an infant; heterogeneous tissue as conjunctiva from the rabbit, first employed by $W_{0} f^{2}$ in 1883. Harlan ${ }^{3}$ has devised a method of operating in which by means of a lead wire he seeks to establish a canal at a depth in the tissue corresponding to the normal location of the fornix conjunctivæ. When the canal is lined with epithelium throughout, the tissue separating the margins of the lids from the canals is divided and by means of a lead shell so shaped that the borders rest in the canals, the raw surfaces are kept from uniting.

\section{SHRINKAGE IS THE CAUSE OF FAILURE.}

All of the procedures heretofore devised have resulted in frequent failures; partial success has attended all, but none give sufficient assurance of being successful. Failures result, not from sloughing of the transplanted flap, as even the heterogeneous flaps usually "take," but from subsequent shrinking, which results in complete loss of the primary favorable result. Heterogeneous flaps in the great majority of cases disappear almost entirely.

Shrinking takes place in all transplanted flaps, the flap with a pedicle shrinks least - the heterogenous flap most. The Thiersch graft shrinks more than the Wolfe flap. The writer has found that the Wolfe flap, placed on a surface of loose yielding tissue and not attached to a fixed border, shrinks to about one-fifth of its original area and becomes much thickened. Shrinking of transplanted flaps is less extensive if the margins of the flap can be attached to fixed points where they can be secured for some weeks. Thus in operating for the relief of excessive ectropion the margins of the lids, when practicable, are united in three or four places and the defect to be covered is made as large as possible by drawing the lids upward, in operations for ectropion of the lower lid, and fastening the sutures, which are left

* Read at the Fifty-third Annual Meeting of the American Medical Association, in the Section on Ophthalmology, and approved for publication by the Executive Committee: Drs. Frank Allport, U. V. Würdemann and J. A. Lippincott.

1. Cirincione: La Clinica Ocolistica, March, 1902, p. 868.

2. Wolfe: Orig. Contributions to Ophth. Surg., London, 1890.

3. Harlan: Trans. Amer. Ophthal. Soc., 1897, p. 63. long: to the forehead by means of rubber adhesive plaster; or downward in operating on the upper lid, making the attachment to the cheek. This excessive enlargement of the defect should be maintained for a period of two or three weeks. The union between the margins of the lids should be permitted to remain for two or three months or until the shrinking is about complete. In this manner margins more or less fixed are obtained and the shrinkage is reduced to a minimum. The tissue of the orbit and lids is particularly non-resisting. Flaps placed on this tissue, if not attached to points more or less fixed, shrink excessively. If a shell of any kind be worn to maintain a cul-de-sac on the transplantation of a Hap, the sac may be maintained for some months, but eventually the sac will become greatly reduced and in many cases will entirely disappear.

The period of shrinking of the Wolfe flap is, as a rule, about three months; of this fact the writer has assured himself by repeated measurements of many transplanted flaps. The area obtained is seldom more than one-fourth the area of the flap before it is removed from its place of origin, and more often one-fifth of the original area is the extent of the permanent surface.

DETAILS OF TIIE AUTIIOR'S METIIOD.

The operation that the writer has performed with unvarying success is as follows: One cul-de-sac only is rectored at one operation for the reason that if sloughing shcald occur the work of restoring one sac only would be destroyed; also because the time required to perform two operations would necessitate an excessively prolonged anesthesia.

The area of operation is rendered as nearly aseptic as possible. Ether anesthesia is necessary. The lid, upper or lower, as the case may be, is dissected from the orbital tissue, leaving the greater part of the conjunctiva (if conjunctiva is present) on the orbital surface. The tissue attached to the lid should be no more than enough to include the fibers of the orbicularis palpebrarum muscle; the dissection should be carried downward to the tissue just above the periosteum at the margin of the orbit. If the palpebral fissure is shorter than normal or if the margin of the lid is tense and the dissection, in forming the groove, is hampered, a free canthotomy should be made. The groove, or sac formed by the dissection, may be carried outward so as to include the portion exposed by the canthotomy. Having completed the groove, bleeding. which is little more than oozing from small vessels, is controlled by packing with aseptie absorbent gauze. A protective pad is applied.

The thin skin on the inner aspect of the arm is selected for the flap. By measurements quickly made the size and shape of the flay can be determined. The area of the flap before removal should be at least one-third larger than the defect to be covered. The area from which the flap is taken should be rendered aseptic some hours before the operation and an aseptic dressing applied. In removing the flap care should be observed to remove the skin only, if subcutaneous tissue to any amount is detached the flap will be too thick. After the flap is separated from the arm it is placed in a warm, sterile, normal saline solution on a piece of plain absorbent gauze. Should subcutaneous tissue be found on the flap it should be trimmed off. This can be done by means of a pair of curved scissors, the flap being stretched over the thumb or index finger. The flap is now folded with epithelial surfaces in apposition. Three long sutures of No. 5 black silk, each armed with two needles are now passed through the flap at the bottom of the fold, so that each suture forms a loop on the epithelial 